\title{
Microscope for quantum dynamics with Planck cell resolution
}

\author{
Zhenduo Wang (王朕铎) $\odot,{ }^{1}$ Jiajin Feng (冯嘉进) $\odot,{ }^{1}$ and Biao Wu (吴胶) ${ }^{1,2,3}$ \\ ${ }^{1}$ International Center for Quantum Materials, School of Physics, Peking University, Beijing 100871, China \\ ${ }^{2}$ Wilczek Quantum Center, School of Physics and Astronomy, Shanghai Jiao Tong University, Shanghai 200240, China \\ ${ }^{3}$ Collaborative Innovation Center of Quantum Matter, Beijing 100871, China
}

(Received 17 February 2021; accepted 17 August 2021; published 10 September 2021)

\begin{abstract}
We introduce the out-of-time-correlation (OTOC) with the Planck cell resolution. The dependence of this OTOC on the initial state makes it function like a microscope, allowing us to investigate the fine structure of quantum dynamics beyond the thermal state. We find an explicit relation of this OTOC to the spreading of the wave function in the Hilbert space, unifying two branches of the study of quantum chaos: state evolution and operator dynamics. By analyzing it in the vicinity of the classical limit, we clarify the dependence of the OTOC's exponential growth on the classical Lyapunov exponent.
\end{abstract}

DOI: 10.1103/PhysRevResearch.3.033239

\section{INTRODUCTION}

In recent years, the out-of-time-order correlation (OTOC) $[1,2]$ has attracted great attention in the field of quantum dynamics [3,4]. It offers us a powerful tool to quantify quantum chaotic behavior, particularly, in many-body systems [5-7]. However, there remains some fundamental issues, two of which are what we try to resolve in this paper.

The cause of the growth of the OTOC is not clear yet. It has been found that the early-time growth of the OTOC is related to the classical Lyapunov exponent [8-12]. This makes the OTOC popular in the study of quantum chaos. However, recent works demonstrated such exponential growth can be caused by a saddle point but not chaos $[13,14]$. One of the reasons is that the usual OTOC [2] (thermal OTOC below) has no dependence on the initial conditions, which is a general feature of all dynamics. In particular, as is well known, the dynamics of the same classical system can be regular for one set of initial conditions and chaotic for another set of initial conditions. This is usually illustrated with the Poincaré section [e.g., see Fig. 1(a)] [15-17]. Thus, we cannot directly use the growth of the OTOC as an indicator to classify dynamics.

Quantum chaotic behavior can also be characterized by the wave-packet spreading [18-24]. This Schrödinger picture dynamics corresponds to our intuitive understanding to chaotic motion. In contrast, the OTOC reflects the quantum dynamics in the Heisenberg picture [23,25]. Some evidence implies that these two pictures are related [4,23], but an analytical derivation is still lacking.

Published by the American Physical Society under the terms of the Creative Commons Attribution 4.0 International license. Further distribution of this work must maintain attribution to the author(s) and the published article's title, journal citation, and DOI.
To resolve the above issues, we introduce a modified version of the OTOC,

$$
C(t, x)=-\left\langle x\left|[\hat{Q}(t), \hat{P}(0)]^{2}\right| x\right\rangle .
$$

State $|x\rangle$ for one dimension is

$$
|x\rangle \equiv\left|Q_{x}, P_{x}\right\rangle=\frac{1}{\sqrt{\Delta_{q}}} \int_{Q_{x}}^{Q_{x}+\Delta_{q}} d q|q\rangle e^{i P_{x} q / \hbar} .
$$

The generalization to higher dimensions is straightforward. Variable $x$ traverses all Planck cells [squares in Fig. 1(b)] by $x=\left(Q_{x}, P_{x}\right)=x_{0}+\left(m \Delta_{q}, n \Delta_{p}\right)$ where $m, n$ are integers, $\Delta_{q}, \Delta_{p}$ are size of cells along $q, p$ axes with $\Delta_{q} \Delta_{p}=2 \pi \hbar$, and $x_{0}$ is the origin of phase space. These states $|x\rangle$ form a set of complete orthonormal basis (see Appendix A), and they are localized in both position and momentum space [26-28]. Operators $\hat{Q}=\sum_{x}|x\rangle Q_{x}\langle x|$ and $\hat{P}=\sum_{x}|x\rangle P_{x}\langle x|$ are so-called macroscopic position and momentum operators [21,28-30]. There are already many variations of the OTOC $[2,3,9,31,32]$. Compared to these definitions and the original one [1,2], our definition of the OTOC is state dependent. That is, for different states $|x\rangle$, this OTOC has distinct longtime behavior. As a result, we can use it to plot a quantum version of the Poincaré section. An example of the quantum kicked rotor [26,27] is shown in Fig. 1(b), which captures the salient features in the corresponding classical Poincaré section in Fig. 1(a). Since the thermal OTOC [2] averages over all quantum states, our OTOC functions, such as a microscope for quantum dynamics with Planck cell resolution. Furthermore, we can analytically show that the dynamical behavior of this OTOC is explicitly related to the wave packet spreading and clarify the dependence of the OTOC growth on the classical Lyapunov exponent.

\section{TIME EVOLUTION OF THE OTOC}

The long-time behavior of the OTOC in Eq. (1) can be shown related to the wave packet spreading explicitly. To see this, we consider the semiclassical limit $\hbar \rightarrow 0$. At this limit, 

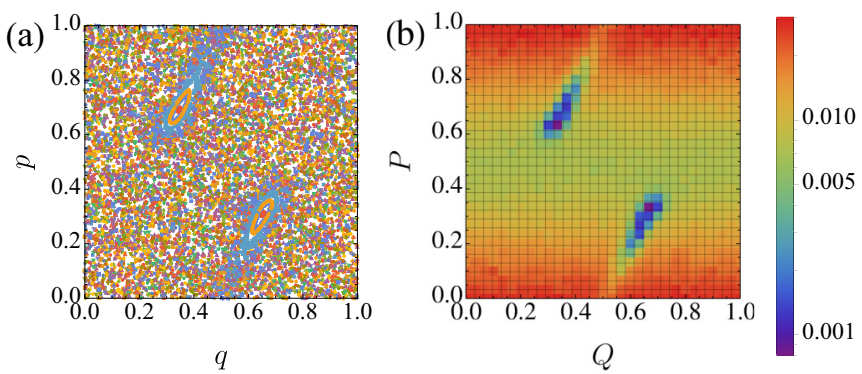

FIG. 1. The comparison between the classical Poincaré section and its quantum version for a kicked rotor. (a) The classical one is generated by random sampling in phase space and with 100 kicks. (b) The quantum version with dimensionless effective Planck constant $\hbar \approx 0.007$ is constructed by computing the OTOC defined in Eq. (1) for each Planck cell with 70 kicks. The blue valleys of the OTOC correspond to the classical integrable islands. The kicking strength is $K=4.7$.

the matrix elements of the propagator $\left\langle q^{\prime}|\hat{U}| q\right\rangle \sim e^{\mathrm{i} S\left(q, q^{\prime}\right) / \hbar}$ [33] in the basis of Planck cells can be written as

$$
\begin{aligned}
\left\langle Q^{\prime}, P^{\prime}|\hat{U}| Q, P\right\rangle \propto & \int_{Q^{\prime}}^{Q^{\prime}+\Delta_{q}} d q^{\prime} \int_{Q}^{Q+\Delta_{q}} d q \\
& \times \exp \left(i \frac{P q-P^{\prime} q^{\prime}+S\left(q, q^{\prime}\right)}{\hbar}\right) .
\end{aligned}
$$

Because of the stationary-phase approximation [34], the nonvanishing zeroth-order term of the above integral is determined by equations,

$$
P=-\frac{\partial S\left(Q, Q^{\prime}\right)}{\partial Q}, \quad P^{\prime}=\frac{\partial S\left(Q, Q^{\prime}\right)}{\partial Q^{\prime}},
$$

which is the classical trajectory governed by action $S$ [15]. This suggests us to rewrite Eq. (3) as follows:

$$
\left\langle x^{\prime}|\hat{U}(t)| x\right\rangle=e^{i \phi(x)} \delta_{x^{\prime}, g_{c} x}+f\left(x^{\prime}, x, t\right),
$$

where $x, x^{\prime}$ are Planck cells as in Eq. (2) and $g_{c} x$ is the cell containing $g x$, which is the state driven classically beginning from $x$ at time $t$. The function $f$, which we call the quantum spreading function, describes the pure quantum dynamics on top of the classical dynamics. When $\hbar$ gets to zero, the function $f$ vanishes and Planck cells become continuous such that $g_{c}=g$. In this limit, the pure quantum dynamics is completely suppressed, and we are left with only the classical dynamics.

The left-hand side of Eq. (5) $\left\langle x^{\prime}|\hat{U}(t)| x\right\rangle$ describes how a wave packet initially localized at Planck cell $x$ evolves and arrives at Planck cell $x^{\prime}$. The right-hand side shows that it has two different contributions. The first term transports dynamically state $x$ into its classical target $g x$. Due to the finite size of Planck cells, $g_{c}$ implements a coarse-grained version of classical dynamics. When the evolution time is shorter or around Ehrenfest time [35,36], this term dominates. The second term breaks this classical picture and depicts how widely the wave packet spreads due to quantum effects. The function $f\left(x^{\prime}, x, t\right)$ begins to be significant after the Ehrenfest time and becomes dominate beyond another timescale called quantum time [37]. In the following, we show how they contribute separately to the OTOC and result in quantum chaos.
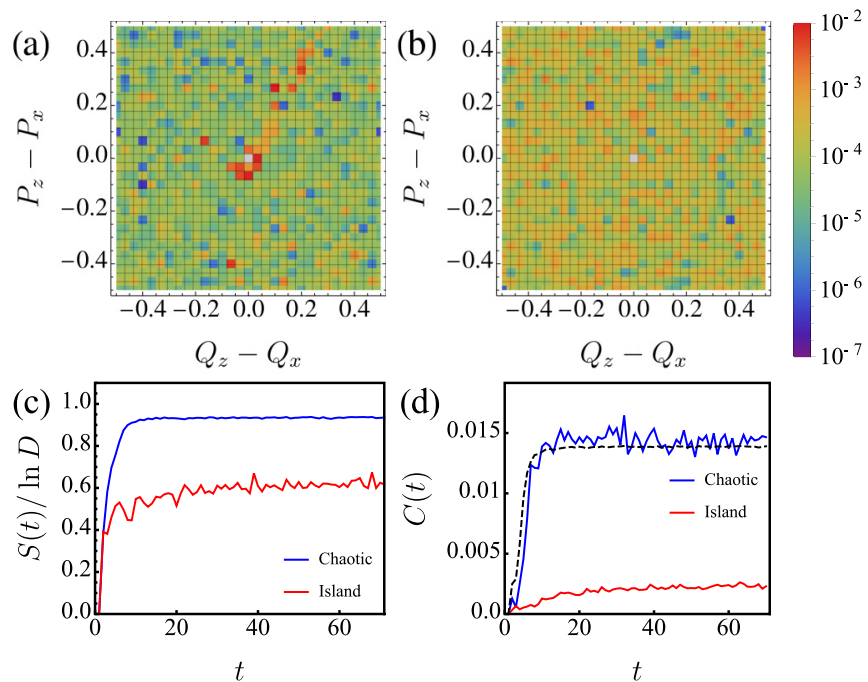

FIG. 2. The value of $\left|f\left(g_{c} z, x, t\right)\right|^{2}$ at points $z-x=\left(Q_{z}-\right.$ $\left.Q_{x}, P_{z}-P_{x}\right)$ for the kicked rotor of kicking strength $K=4.7$. The number of kicks is 40 , and the effective Planck constant $\hbar \approx 0.007$. The initial state for (a) is the Planck cell $x=(0.35,0.7)$ (inside an integrable island); the initial state for (b) is $x=(0.2,0.2)$ (inside the chaotic sea). They are also, respectively, the initial states for the island results and chaotic in (c) and (d). (c) The growth of normalized GWvN entropy [38]. (d) The growth of the OTOC. The dashed line is the thermal OTOC at temperature $T=\infty$ as in Eq. (7).

With Eq. (5) we can show an explicit relation between the growth of the OTOC and the wave-packet diffusion. The leading order of the OTOC in Eq. (1) is the second order of $f$ and can be written as (see Appendix B),

$$
C(t, x)=\sum_{z}\left(P_{z}-P_{x}\right)^{2}\left(Q_{g_{c} z}-Q_{g_{c} x}\right)^{2}\left|f\left(g_{c} z, x, t\right)\right|^{2},
$$

where $\left(Q_{x}, P_{x}\right)$ are the coordinate and momentum of the Planck cell $|x\rangle$. The time-dependent terms are $\left(Q_{g_{c} z}-Q_{g_{c} x}\right)^{2}$ and $\left|f\left(g_{c} z, x, t\right)\right|^{2}$. The former is the partial distance between trajectories. It can reflect the sensibility of the coarse-grained classical dynamics $g_{c}$ to the initial condition $x$ when $z$ is close to $x$. The latter one weights these terms in this summation. The region around $x$ in which $\left|f\left(g_{c} z, x, t\right)\right|^{2}$ is significant shows how widely the wave packet spreads.

The features of the quantum Poincaré section shown in Fig. 1(b) are due to the function $\left|f\left(g_{c} z, x, t\right)\right|^{2}$. For $x$ initially in a classical integrable island, $\left|f\left(g_{c} z, x, t\right)\right|^{2}$ cannot spread as widely as it does in chaotic sea. This is clearly demonstrated by the numerical results in Figs. 2(a) and 2(b). For point $x$ located in an integrable island, function $\left|f\left(g_{c} z, x, t\right)\right|^{2}$ is significant only when $z$ is close to $x$. This together with the regular motion in an integrable island makes OTOC $C(t, x)$ saturate at a small value: $\left(P_{z}-P_{x}\right)^{2}$ measures the size of this small region, whereas $\left(Q_{g_{c} z}-Q_{g_{c} x}\right)^{2}$ cannot grow fast because of the regular motion. In contrast for point $x$ located in a chaotic sea, $\left|f\left(g_{c} z, x, t\right)\right|^{2}$ widely spreads over the chaotic sea and renders a larger saturation value for the OTOC. In addition, we use the generalized Wigner-von Neumann entropy [38] to show how widely the wave packet spreads in the long run in Fig. 2(c). In Fig. 2(d), our OTOC behaves 

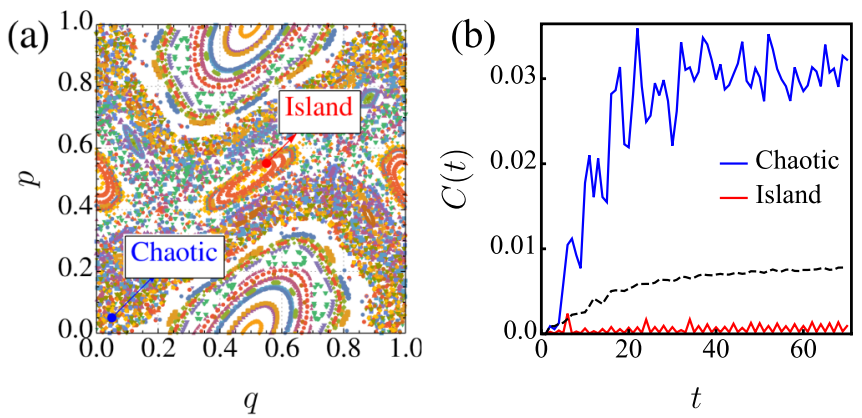

FIG. 3. (a) The Poincaré section of the classical kicked rotor of kicking strength $K=1.3$. (b) The time evolution of our OTOC for two Planck cell states corresponding to the labeled points in (a). For comparison, the thermal OTOC at temperature $T=\infty$ is also computed (dashed line).

similarly. This further confirms that our OTOC is related to the wave-packet spreading.

The thermal OTOC cannot illustrate the state dependency of dynamics, such as the quantum Poincaré section in Fig. 1(b). It computes the expectation value for the thermal state at infinitely high temperature as

$$
\bar{C}(t)=-\frac{\operatorname{Tr}\left[\hat{U}^{\dagger} \hat{Q} \hat{U}, \hat{P}\right]^{2}}{\operatorname{Tr} \mathbb{1}}=\frac{1}{D} \sum_{x} C(t, x),
$$

where $D=\operatorname{Tr} \mathbb{1}$ is the dimension of the Hilbert space and is also the number of Planck cells. We note that it agrees with the thermal state whose density matrix is proportional to identity as $\hat{\rho}=\mathbb{1} / \operatorname{Tr} \mathbb{1} . \bar{C}(t)$ is the average of $C(t, x)$ over the whole phase space or all the Planck cells with uniform weight. The behavior of $\bar{C}(t)$ is determined by the sizes of the areas occupied by integrable islands and chaotic seas in phase space. In Fig. 2(d), the thermal OTOC behaves, such as the chaotic case (blue line) because the chaotic sea dominates. As a comparison, we consider a kicking strength that is close to $K_{c} \sim 0.972$ [39]. The areas of integrable islands and chaotic sea are balanced as shown in Fig. 3(a). This leads to the dynamics in Fig. 3 (b) in which $\bar{C}(t)$ is different from $C(t, x)$ for both integrable and chaotic cases. If we regard the OTOC as a microscope imaging the dynamical structure in the phase space, our OTOC in Eq. (1) has Planck cell resolution, which is the best allowed by quantum mechanics, whereas the thermal one, namely, $\bar{C}(t)$ can only render a smeared image with the crudest resolution that can only reveal whether the dominant part is an integrable island or a chaotic sea.

In this section, we have achieved two goals mentioned in the Introduction. First, we provide an OTOC-like tool to study the dynamics with initial condition dependence by Eq. (1) with Planck cell resolution. Second, we answer how two pictures are related in quantum chaos with Eq. (6). It analytically attributes the growth of the OTOC to the coarse-grained classical dynamics and wave-packet spreading. The former one is numerically studied in Ref. [4,23], whereas the latter one, which is affected by the energy spectrum, has been discussed $[40,41]$. In the next section, we compare our OTOC with the common one in the vicinity of classical dynamics, showing the advantage of our form.

\section{THE OTOC AND LYAPUNOV EXPONENT}

The early-time growth of our OTOC is related to the classical Lyapunov exponent. The quantum spreading function $f\left(x^{\prime}, x, t\right)$ is very narrow during the early time evolution no matter whether $x$ is located in the integrable islands or chaotic sea. As a result, we are allowed to take $f$ out from the summation in Eq. (6) and obtain

$$
C(t, x) \approx A \sum_{z}\left(Q_{g_{c} z}-Q_{g_{c} x}\right)^{2} \sim e^{2 \lambda Q^{t},}
$$

where $A=\overline{\left(P_{z}-P_{x}\right)^{2}\left|f\left(g_{c} z, x\right)\right|^{2}}$ is a positive number and the summation is over the neighborhood of $x$. The last term with $\lambda_{Q}$ is due to that $\left(Q_{g_{c} z}-Q_{g_{c} x}\right)^{2}$ is the result of the coarse-grained classical dynamics. At the limit of $\hbar \rightarrow 0$, the Lyapunov exponent of OTOC $\lambda_{Q}$ becomes the classical Lyapunov exponent $\lambda_{C}$ because of $\lim _{\hbar \rightarrow 0} g_{c}=g$. When time $t$ is not short and/or the Planck constant $\hbar$ is not small enough, the factor $|f|^{2}$ cannot be taken out of the summation. Therefore, the exponential growth of the OTOC is not guaranteed, in general, because the evolution of $|f|^{2}$ is influenced not just by the classical Lyapunov exponent.

To demonstrate the exponential growth in Eq. (8) numerically, we turn to the continuous time evolution in a two-site Bose-Hubbard model [42-44]. There are two reasons for this choice: (1) This model is simple enough for a reliable numerical study; (2) the time evolution of the kicked rotor is discretized and not convenient for analyzing short-time behavior. See Appendix C for the chaotic but discretized case. That makes our result more robust. This Bose-Hubbard model is also studied in Ref. [13] as the Lipkin-Meshkov-Glick (LMG) model. Its Hamiltonian is given by (with $\hbar=1$ ) [44]

$$
\hat{H}=\frac{1}{2}\left(\hat{a}_{1}^{\dagger} \hat{a}_{0}+\hat{a}_{0}^{\dagger} \hat{a}_{1}\right)-\frac{1}{N}\left(\hat{a}_{1}^{\dagger} \hat{a}_{1}-\hat{a}_{0}^{\dagger} \hat{a}_{0}\right)^{2},
$$

where $\hat{a}_{0,1}, \hat{a}_{0,1}^{\dagger}$ are annihilation and creation operators and $N$ is the total number of bosons in the system. For this system, the effective Planck constant is $1 / N$. When $N \rightarrow$ $\infty$, the system becomes classical in the sense of mean-field approximation $[45,46]$. The system can be described by a single-particle Hamiltonian system of

$$
H(q, p)=\sqrt{1 / 4-p^{2}} \cos q-4 p^{2} .
$$

There is a saddle point at $x^{*}=\left(q^{*}, p^{*}\right)=(\pi, 0)$, and the Lyapunov exponent of it is $\lambda_{C}=\sqrt{3}$ (see Appendix D).

We focus on the quantal system of finite but large $N$. The Planck cell basis of discretized form (see Appendix D 1) is defined with the eigenstates of momentum operator $\hat{p}=$ $\left(\hat{a}_{0}^{\dagger} \hat{a}_{0}-\hat{a}_{1}^{\dagger} \hat{a}_{1}\right) / 2 N$ [37,44]. In Fig. 4(a), the growth of the OTOC of $C(t)=\left\langle x^{*}\left|[\hat{P}(t), \hat{P}(0)]^{2}\right| x^{*}\right\rangle$ at the saddle point is shown. It is clear from the fitting that the growth of $C(t)$ is exponential with the double of classical Lyapunov exponent of $2 \sqrt{3}$, which agrees with our analysis in Eq. (8). As a comparison, we have also computed the OTOC in the form of $-\left\langle[\hat{p}(t), \hat{p}(0)]^{2}\right\rangle$, which was discussed in Ref. [13]. Our computation is performed both at infinity temperature and on the Planck cell of the saddle point. The results are shown in Fig. 4(b). Although we also see exponential growth, however, both exponents are different from the classical Lyapunov exponent. We also plot the growth of the width $W^{2}(t)$ of the 

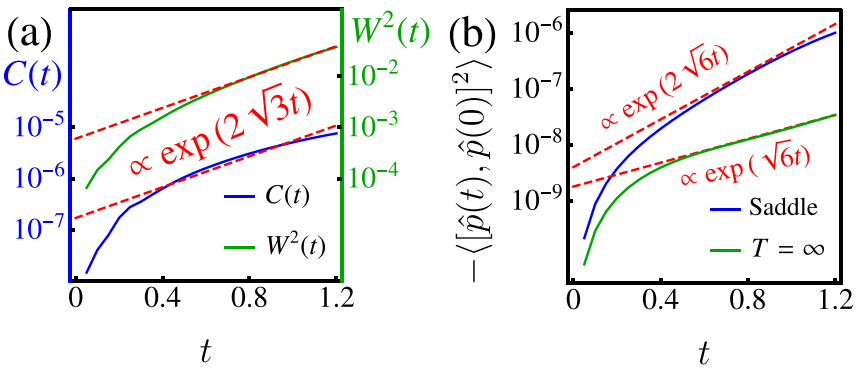

FIG. 4. Numerical results of the LMG model of $N=1681$ and $\hbar_{\text {eff }} \sim 1 / N=0.0005$. (a) Our OTOC with macroscopic momentum $\hat{P}$ at the saddle point (blue line). The green line is the width of the wave packet along the $P$-axis $W^{2}(t)$. Two red lines are both of exponent $2 \sqrt{3}=\lambda_{C}$. (b) The OTOC with momentum $\hat{p}$. The blue line is for its expectation value over $\left|x^{*}\right\rangle$, the Planck cell basis of the classical saddle point; the green line is the usual expectation over the thermal state at $T=\infty$.

wave function along axis of $P$ in Fig. 4(a). It is an approximation to Eq. (6) by

$$
\begin{aligned}
W^{2}(t) & =\left\langle x^{*}\left|\hat{U}^{\dagger}(\hat{P}(0)-0)^{2} \hat{U}\right| x^{*}\right\rangle \\
& \approx \sum_{z}\left(P_{g_{c} z}-P_{g_{c} x^{*}}\right)^{2}\left|f\left(g_{c} z, x^{*}\right)\right|^{2} \sim C(t) .
\end{aligned}
$$

We can find that its growth also fits the classical Lyapunov exponent well.

Note that we have replaced $Q$ with $P$ in Eq. (6) in the above computation for the convenience of comparison to the results in Ref. [13]. Technically, since $\hat{Q}, \hat{P}$ commute, our results for the OTOC in Eq. (6) can be generalized for any operator pairs that are functions of $\hat{Q}, \hat{P}$ as

$$
\begin{aligned}
C_{A B}(t, x)= & \left.-\langle x|\left[\hat{U}^{\dagger} A(\hat{Q}, \hat{P})\right] \hat{U}, B(\hat{Q}, \hat{P})\right]^{2}|x\rangle \\
= & \sum_{z}\left[A\left(g_{c} z\right)-A\left(g_{c} x\right)\right]^{2}[B(z)-B(x)]^{2} \\
& \times\left|f\left(g_{c} z, x, t\right)\right|^{2},
\end{aligned}
$$

where $A, B$ are functions defined in phase space.

As indicated by the results in Fig. 4, the exponent of the OTOC can be different from the classical Lyapunov exponent. This difference has been noted before [9,47]. Our numerical result shows that it is deeply related to the choice of operators. The OTOC with $\hat{p}$ at the saddle point [the blue line in Fig. 4(b)] grows different from classical Lyapunov exponent, whereas our OTOC with $\hat{P}$ does not.

A probable explanation for the inconsistency is that the classical limit for the LMG model is different from systems with spatial degrees of freedom. There is no canonical commutation relation of $[\hat{q}, \hat{p}]=i \hbar$ in the finite-dimensional Hilbert space. The classical dynamics of the LMG model is described as the transportation among Planck cell basis [the zeroth order in Eq. (5)]. Since Eq. (6) only relies on this property of quantum evolution, our OTOC and the analysis still work for the LMG model. It is shown in Ref. [12] that under the Wigner-Weyl transformation, the operator $-[\hat{q}(t), \hat{p}(0)]^{2}$ leads to a function characterized by classical Lyapunov exponent in phase space in the classical limit. We illustrate the thermal OTOC of inverted harmonic oscillator [14] in
Appendix E. In this system with intrinsic symplectic form, exponential growth of the OTOC of $\hat{q}$ and $\hat{Q}$ shares the same exponent and agrees with the classical value.

\section{CONCLUSION}

We have introduced a state-dependent form of the OTOC, which serves as a microscope for resolving the fine structure in quantum dynamics. With analytical derivation, we have extracted and explained two sources of the growth of this OTOC. One controls its short-time behavior whereas the other determines its long-time saturation. The early-time exponential growth of the OTOC is caused by the former and related to the classical Lyapunov exponent. We are also able to show explicitly how the operator correlation in the OTOC is related to wave-packet spreading in Hilbert space.

\section{ACKNOWLEDGMENTS}

This work was supported by the the National Key R\&D Program of China (Grants No. 2017YFA0303302 and No. 2018YFA0305602), the National Natural Science Foundation of China (Grant No. 11921005), and the Shanghai Municipal Science and Technology Major Project (Grant No. 2019SHZDZX01).

\section{APPENDIX A: BASIS OF QUANTUM PHASE SPACE}

We focus on a one-dimensional system; the results can be generalized straightforwardly to higher dimensions. For a one-dimensional classical system, its phase pace is two dimensional. In quantum statistical mechanics, one usually divides the phase space into Planck cells to obtain quantum phase space. In 1929, von Neumann proposed to construct a set of orthonormal basis by assigning each Planck cell a localized wave function $[29,30]$. This idea has been further developed with the help of the Wannier functions [21,26,28].

One basis for such a quantum phase space can be constructed with the following basis function:

$$
|Q, P\rangle=\frac{1}{\sqrt{\Delta_{q}}} \int_{Q}^{Q+\Delta_{q}} d q|q\rangle e^{i P q / \hbar} .
$$

Here $q$ is the position of a particle, and $|q\rangle$ is the eigenstate of $\hat{q} . \Delta_{q}$ is one side of the Planck cell, and the other side is $\Delta_{p}$ such that $\hat{q} . \Delta_{q}$ 's are discretized position and momentum of a given Planck cell.

They are orthonormal: $\left\langle Q^{\prime}, P^{\prime} \mid Q, P\right\rangle=\delta_{Q, Q^{\prime}} \delta_{P, P^{\prime}}$. Here is the proof,

$$
\begin{aligned}
& \left\langle Q^{\prime}, P^{\prime} \mid Q, P\right\rangle \\
& =\frac{1}{\Delta_{q}} \int_{Q}^{Q+\Delta_{q}} \int_{Q^{\prime}}^{Q^{\prime}+\Delta_{q}} d q d q^{\prime} \delta\left(q-q^{\prime}\right) e^{i P q / \hbar-i P^{\prime} q^{\prime} / \hbar} \\
& =\frac{1}{\Delta_{q}} \delta_{Q, Q^{\prime}} \int_{Q}^{Q+\Delta_{q}} d q e^{i\left(P-P^{\prime}\right) q / \hbar} \\
& =\delta_{Q, Q^{\prime}} \frac{e^{i\left(P-P^{\prime}\right) Q / \hbar}}{i\left(P-P^{\prime}\right) / \hbar}\left[e^{i\left(P-P^{\prime}\right) \Delta_{q} / \hbar}-1\right] \\
& =\delta_{Q, Q^{\prime}} \delta_{P, P^{\prime}},
\end{aligned}
$$


where we have used the property that $P-P^{\prime} \equiv 0 \bmod \Delta_{p}$. That they are normalized can also be checked.

We can also construct the Planck cell basis with eigenstates of $\hat{p}$ as

$$
|Q, P\rangle_{m}=\frac{1}{\sqrt{\Delta_{p}}} \int_{P}^{P+\Delta_{p}} d p|p\rangle e^{-i Q p / \hbar} .
$$

The sign difference in the exponent comes from the symplectic structure of classical mechanics. Only when we use such a sign for $Q p$, we can get the correct classical equation of motion from the quantum propagator at the limit of $\hbar \rightarrow 0$. This set of basis is consistent with the previous one, that is,

$$
\lim _{\hbar \rightarrow 0}\left|\left\langle Q^{\prime}, P^{\prime} \mid Q, P\right\rangle_{m}\right|^{2}=\delta_{Q, Q^{\prime}} \delta_{P, P^{\prime}}
$$

One can check it as follows:

$$
\begin{aligned}
\left\langle Q^{\prime}, P^{\prime} \mid Q, P\right\rangle_{m} & \\
= & \frac{1}{\sqrt{\Delta_{q} \Delta_{p}}} \int_{P}^{P+\Delta_{p}} d p \int_{Q^{\prime}}^{Q^{\prime}+\Delta_{q}} d q e^{-i Q p / \hbar-i P^{\prime} q / \hbar}\langle q \mid p\rangle \\
= & \frac{1}{\sqrt{h V}} \int_{0}^{\Delta_{p}} d p \int_{0}^{\Delta_{q}} d q \exp \{-i[Q(p+P) \\
& \left.\left.+P^{\prime}\left(q+Q^{\prime}\right)-(p+P)\left(q+Q^{\prime}\right)\right] / \hbar\right\} \\
= & \frac{e^{i \alpha}}{\sqrt{h V}} \int_{0}^{\Delta_{p}} d p \int_{0}^{\Delta_{q}} d q e^{-i\left[\left(Q-Q^{\prime}\right) p+\left(P^{\prime}-P\right) q-q p\right] / \hbar} .
\end{aligned}
$$

At the limit of $\hbar \rightarrow 0$, the main contribution of the integral appears at the point of $Q=Q^{\prime}, P=P^{\prime}$.
APPENDIX B: MATHEMATICAL DETAILS FOR $C(t, x)$

With $x=\left(Q_{x}, P_{x}\right)$ and operators,

$$
\hat{Q}=\sum_{x}|x\rangle Q_{x}\left\langle x\left|; \hat{P}=\sum_{x}\right| x\right\rangle P_{x}\langle x|,
$$

we have

$$
\begin{aligned}
-C(t, x)= & \left\langle x\left|\left[\hat{U}^{\dagger} \hat{Q} \hat{U}, \hat{P}\right]^{2}\right| x\right\rangle \\
= & \langle x|\left(\sum _ { z , z ^ { \prime } , z ^ { \prime \prime } } | z ^ { \prime \prime } \rangle \langle z ^ { \prime } | \left(\left\langle z^{\prime \prime}\left|\hat{U}^{\dagger}\right| z\right\rangle\left\langle z|\hat{U}| z^{\prime}\right\rangle Q_{z} P_{z^{\prime}}\right.\right. \\
& \left.\left.-\left\langle z^{\prime \prime}\left|\hat{U}^{\dagger}\right| z\right\rangle\left\langle z|\hat{U}| z^{\prime}\right\rangle Q_{z} P_{z^{\prime \prime}}\right)\right)^{2}|x\rangle \\
= & -\sum_{z_{1}, z_{2}, z_{3}}\left\langle x\left|\hat{U}^{\dagger}\right| z_{1}\right\rangle\left\langle z_{1}|\hat{U}| z_{2}\right\rangle\left\langle z_{2}\left|\hat{U}^{\dagger}\right| z_{3}\right\rangle \\
& \times\left\langle z_{3}|\hat{U}| x\right\rangle Q_{z_{1}} Q_{z_{3}}\left(P_{z_{2}}-P_{x}\right)^{2} .
\end{aligned}
$$

Since the propagator reads

$$
\left\langle x^{\prime}|\hat{U}| x\right\rangle=e^{i \phi(x)} \delta_{x^{\prime}, g_{c} x}+f\left(x^{\prime}, x\right),
$$

we can compute $C(t, x)$ by the orders of $f$. The zeroth order of $C$ is made up with four Kronecker symbols. Then the product leads to factor of $\delta_{z_{2}, x}$, this factor together with $\left(P_{z_{2}}-P_{x}\right)^{2}$ makes the zeroth order vanish. For the same reason, the first order of $C$ contains a product of three Kronecker symbols, $z_{2}$ will always be connected with $x$ and contributes a zero factor. Thus, the leading term of $C(t, x)$ should be the second order of $f$. With the omission of two terms in which the product of Kronecker symbols connects $x$ and $z_{2}$, the nonzero terms of the second order are

$$
\begin{aligned}
C^{(2)}= & \sum_{z} f^{*}(z, x) f(z, x) Q_{z}^{2}\left(P_{g_{c}^{-1} z}-P_{x}\right)^{2}+\sum_{z} f\left(g_{c} x, z\right) f^{*}\left(g_{c} x, z\right) Q_{g_{c} x} Q_{g_{c} x}\left(P_{z}-P_{x}\right)^{2} \\
& +\sum_{z} f\left(g_{c} x, z\right) f\left(g_{c} z, x\right) Q_{g_{c} x} Q_{g_{c} z}\left(P_{z}-P_{x}\right)^{2} e^{-i \phi(x)-i \phi(z)} \\
& +\sum_{z} f^{*}(z, x) f^{*}\left(g_{c} x, g_{c}^{-1} z\right) Q_{z} Q_{g_{c} x}\left(P_{g_{c}^{-1} z}-P_{x}\right)^{2} e^{i \phi\left(g_{c}^{-1} z\right)+i \phi(x)} \\
= & \sum_{z}\left(P_{z}-P_{x}\right)^{2}\left|Q_{g_{c} z} f\left(g_{c} z, x\right) e^{-i \phi(x)}+Q_{g_{c} x} f^{*}(g x, z) e^{i \phi(z)}\right|^{2} .
\end{aligned}
$$

With the unitarity of expansion of time evolution operator (up to the first order of $f$ ), we have

$$
\begin{aligned}
\delta_{x, x^{\prime}}= & \sum_{z}\left\langle x^{\prime}|\hat{U}| z\right\rangle\left\langle z\left|\hat{U}^{\dagger}\right| x\right\rangle \\
= & \sum_{z}\left[e^{i \phi(z)} \delta_{x^{\prime}, g_{c} z}+f\left(x^{\prime}, z\right)\right]\left[e^{i \phi(z)} \delta_{x, g_{c} z}+f(x, z)\right]^{*} \\
= & \delta_{x, x^{\prime}}+e^{i \phi\left(g_{c}^{-1} x^{\prime}\right)} f^{*}\left(x, g_{c}^{-1} x^{\prime}\right) \\
& +e^{-i \phi\left(g_{c}^{-1} x\right)} f\left(x^{\prime}, g_{c}^{-1} x\right)+\cdots .
\end{aligned}
$$

This leads to an equality of

$$
e^{i \phi\left(x^{\prime}\right)} f^{*}\left(g_{c} x, x^{\prime}\right)+e^{-i \phi(x)} f\left(g_{c} x^{\prime}, x\right)=0 .
$$

Thus, we have the second order of $C(t, x)$,

$$
C^{(2)}=\sum_{z}\left(P_{z}-P_{x}\right)^{2}\left(Q_{g_{c} z}-Q_{g_{c} x}\right)^{2}\left|f\left(g_{c} z, x\right)\right|^{2} .
$$

This is what we discussed in the main text.

\section{APPENDIX C: EARLY TIME GROWTH OF THE OTOC IN THE KICKED ROTOR}

The dynamics of the kicked rotor is driven by the Hamiltonian of

$$
H=\frac{1}{2} p^{2}+K \cos q \sum_{n=-\infty}^{\infty} \delta(t-n) .
$$



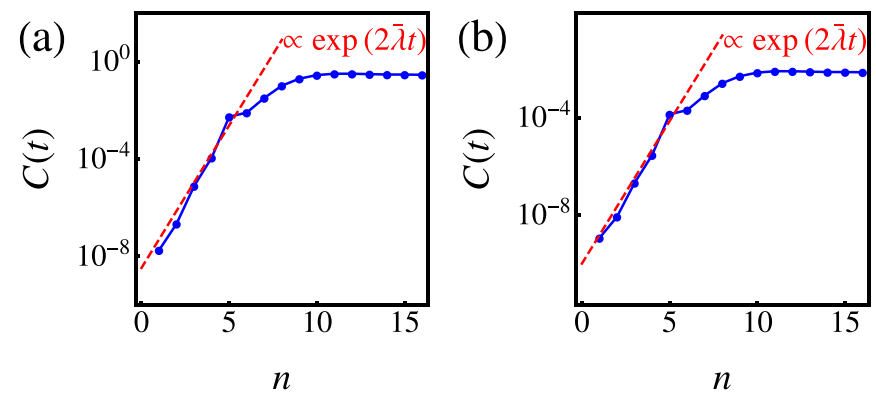

FIG. 5. The value of the OTOC $C(t)$ on the point of $x=(q, p)=$ $(0.5,0.5)$ in the kicked rotor with kicking strength $K=4.7>K_{c} \approx$ 0.97 . The system is chaotic, and the phase-space structure is shown in Fig. 1 in the main text. (a) The growth of the usual OTOC for momentum $C(t)=-\left\langle x\left|[\hat{p}(t), \hat{p}(0)]^{2}\right| x\right\rangle$. The red line is the exponential growth with twice of the average classical Lyapunov exponent $\bar{\lambda} \approx 1.35$. (b) The growth of our OTOC for macromomentum $C(t)=-\left\langle x\left|[\hat{P}(t), \hat{P}(0)]^{2}\right| x\right\rangle$. The red line is the same as the one in (a) (up to a factor).

In classical mechanics with the periodic boundary condition, the dynamics is described by the Chirikov standard map of

$$
\begin{aligned}
& p_{n+1}=p_{n}+K \sin q_{n} \bmod 2 \pi, \\
& q_{n+1}=q_{n}+p_{n+1} \quad \bmod 2 \pi .
\end{aligned}
$$

With proper rescaling of the canonical pair $(q, p) \rightarrow$ $(q / 2 \pi, p / 2 \pi)$, we can confine the dynamics in the region of $[0,1]^{\otimes 2}$. Thus, in the main text our Planck cells are $0 \leqslant$ $Q, P<1$. The dynamics becomes chaotic when the kicking strength $K$ gets beyond the critical value of $K_{c} \sim 63 / 64$ $[27,39]$. Here we present the numerical results on the early growth of the OTOC in this system with $K=4.7$, which are shown in Fig. 5. The parameters used in our computation are the same as the ones used Figs. 1 and 2 in the main text. Similar to what we have performed for the LMG model and bounded inverted harmonic oscillator, we compare two types of the OTOC here. One is the common one with averaged with Planck cell basis $C(t)=-\left\langle x\left|[\hat{p}(t), \hat{p}(0)]^{2}\right| x\right\rangle$; the other is our OTOC $C(t)=-\left\langle x\left|[\hat{P}(t), \hat{P}(0)]^{2}\right| x\right\rangle$. The results are shown, respectively, in Figs. 5(a) and 5(b). The Planck cell basis is $|x\rangle=|Q=0.5, P=0.5\rangle$.

The classical Lyapunov exponent for this system with discretized time domain can be computed with the Jacobian of the map. With the equation of

$$
\left[\begin{array}{l}
\delta p_{n+1} \\
\delta q_{n+1}
\end{array}\right]=\left[\begin{array}{cc}
1 & K \cos 2 \pi q_{n} \\
1 & 1+K \cos 2 \pi q_{n}
\end{array}\right]\left[\begin{array}{l}
\delta p_{n} \\
\delta q_{n}
\end{array}\right],
$$

the Lyapunov at each time can be obtained by the logarithm of eigenvalues of Jacobian. In this case, we take the average largest of Lyapunov during the first six kicks (noting the initial point is at the zeroth kick) is of $\bar{\lambda} \approx 1.35$.

\section{APPENDIX D: LMG MODEL AND ITS MEAN-FIELD THEORY}

We consider a two-mode interacting Bose gas with the following second quantized Hamiltonian:

$$
\hat{H}=\frac{1}{2}\left(\hat{a}_{1}^{\dagger} \hat{a}_{0}+\hat{a}_{0}^{\dagger} \hat{a}_{1}\right)+\frac{\xi}{2 N}\left(\hat{a}_{1}^{\dagger} \hat{a}_{1}-\hat{a}_{0}^{\dagger} \hat{a}_{0}\right)^{2} .
$$

Its mean-field theory can be obtained with the coherent path integral,

$$
U\left(z_{f}^{*}, t_{f} ; z_{i}, t_{i}\right)=\int \mathcal{D} z \mathcal{D} z^{*} e^{i S / \hbar},
$$

in which the action $S=\int_{t_{i}}^{t_{f}} d t\left(i \hbar z^{*} \dot{z}-\langle z|\hat{H}| z\rangle\right)$ with the coherent state $|z\rangle=e^{z_{0} \hat{a}_{0}^{\dagger}+z_{1} \hat{a}_{1}^{\dagger}}|0\rangle . S / N$ should be a $O(1)$ quantity; we then rewrite the propagator as $U=\int e^{i(N / \hbar)(S / N)}$. Obviously the effective Planck constant is $\hbar / N$. Then with the substitution $z \rightarrow x \sqrt{N}$ and letting $\hbar=1$, the mean-field equation of motion can be obtained with the method of steepest gradient (note the constraint of $\left|x_{0}\right|^{2}+\left|x_{1}\right|^{2}=1$ ),

$$
i \frac{d}{d t}\left[\begin{array}{l}
x_{0} \\
x_{1}
\end{array}\right]=\frac{1}{\sqrt{N}} \frac{\delta}{\delta z^{*}}\langle z|\hat{H}| z\rangle=\left[\begin{array}{cc}
\xi\left(\left|x_{0}\right|^{2}-\left|x_{1}\right|^{2}\right)+\xi / 2 N & 1 / 2 \\
1 / 2 & -\xi\left(\left|x_{0}\right|^{2}-\left|x_{1}\right|^{2}\right)+\xi / 2 N
\end{array}\right]\left[\begin{array}{l}
x_{0} \\
x_{1}
\end{array}\right] .
$$

For a mean-field state $\left(x_{0}, x_{1}\right)$, its corresponding quantum state is

$$
\left|\Psi\left(x_{0}, x_{1}\right)\right\rangle=\frac{1}{\sqrt{N !}}\left(x_{0} \hat{a}_{0}^{\dagger}+x_{1} \hat{a}_{1}^{\dagger}\right)^{N}|0\rangle .
$$

With the transformation of $p=\frac{1}{2}\left(\left|x_{0}\right|^{2}-\left|x_{1}\right|^{2}\right), q=$ $\arg x_{1}-\arg x_{0}$, one can find that this system is a Hamiltonian system with Hamiltonian (up to the order of $1 / N$ ),

$$
H(q, p)=\sqrt{1 / 4-p^{2}} \cos q+2 \xi p^{2} .
$$

The saddle point appears at $\left(q^{*}, p^{*}\right)=(\pi, 0)$ when $\xi=$ -2 , and the classical Lyapunov exponent can be determined by linearizing the canonical equation $\dot{q}=\partial_{p} H, \quad \dot{p}=-\partial_{q} H$ near the saddle point,

$$
\left[\begin{array}{l}
\dot{\delta} q \\
\dot{\delta} p
\end{array}\right]=\left[\begin{array}{cc}
\frac{\partial^{2} H}{\partial q \partial p} & \frac{\partial H}{\partial p^{2}} \\
-\frac{\partial^{2} H}{\partial q^{2}} & -\frac{\partial^{2} H}{\partial q \partial p}
\end{array}\right]\left[\begin{array}{l}
\delta q \\
\delta p
\end{array}\right] .
$$

The matrix is

$$
\left.\left[\begin{array}{cc}
\frac{\partial^{2} H}{\partial q \partial p} & \frac{\partial H}{\partial p^{2}} \\
-\frac{\partial^{2} H}{\partial q^{2}} & -\frac{\partial^{2} H}{\partial q \partial p}
\end{array}\right]\right|_{(q, p)=(\pi, 0)}=\left[\begin{array}{cc}
0 & 4 \xi+2 \\
-1 / 2 & 0
\end{array}\right]
$$

which has the spectrum of $\pm \sqrt{3}$ when $\xi=-2$, that is, our Lyapunov exponent for this saddle point.

The operator corresponding to $p$ is

$$
\hat{p}=\left(\hat{a}_{0}^{\dagger} \hat{a}_{0}-\hat{a}_{1}^{\dagger} \hat{a}_{1}\right) / 2 N .
$$

Its eigenstates is of $|s, N-s\rangle=\frac{1}{\sqrt{s !(N-s) !}} \hat{a}_{0}^{\dagger s} \hat{a}_{1}^{\dagger N-s}|0\rangle$ with

$$
\hat{p}|s, N-s\rangle=-\frac{N-2 s}{2 N}|s, N-s\rangle .
$$


(a)

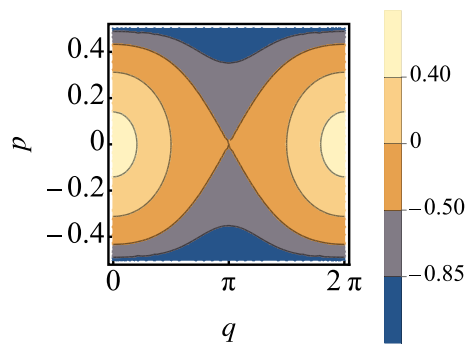

(b)

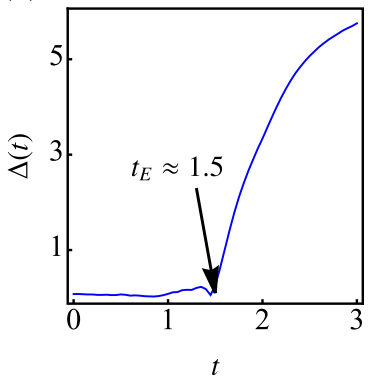

FIG. 6. The LMG model in the classical limit. (a) The energy contours for the classical Hamiltonian in the phase space. (b) The difference between the time evolution for the quantum system at $N=1681$ and the classical one. $\Delta(t)$ measures the $L_{2}$ distance between quantum expectation value of macroscopic operators and the classical trajectory at time $t$.

\section{Phase-space basis for the LMG model}

For systems, such as the LMG model with the finite dimension of the Hilbert space, the definition in Eq. (2) needs to be modified. According to Appendix A, we use eigenstates of $\hat{p}$. The phase space is divided into $L \times L$ cells. The dimension of Hilbert space is $D=N+1=L^{2}$. Letting $L=2 m+1$ be an odd number, the eigenstates of $\hat{p}$ is $\{|p=n / N\rangle\}_{n=-2 m^{2}-2 m}^{2 m^{2}+2 m}$. Then the basis function for the quantum phase-space basis reads

$$
|Q, P\rangle=\frac{1}{\sqrt{L}} \sum_{n=-m+P N}^{m+P N}\left|p=\frac{n}{N}\right\rangle e^{-i N Q p},
$$

with lattice points $(Q, P)=\left(2 \pi n_{1} / L, L n_{2} / N\right)$ in which $n_{1} \in$ $\{0, \ldots, L-1\}$ and $n_{2} \in\{-m,-m+1, \ldots, m\}$. These states are orthonormal,

$$
\begin{aligned}
\left\langle Q^{\prime}, P^{\prime} \mid Q, P\right\rangle & =\frac{1}{L} \sum_{n, n^{\prime}} \delta_{n, n^{\prime}} \exp \left[-i N\left(\frac{Q n}{N}-\frac{Q^{\prime} n^{\prime}}{N}\right)\right] \\
& =\frac{1}{L} \delta_{P, P^{\prime}} \sum_{n=-m+P N}^{m+P N} e^{-i n\left(Q-Q^{\prime}\right)} \\
& =\delta_{P, P^{\prime}} \delta_{Q, Q^{\prime}}
\end{aligned}
$$

Since the effective Planck constant is proportional to $1 / N$ for this system, there is also the classical limit, such as Eq. (4).

\section{Classical limit of the LMG model}

The energy contour plot of the classical Hamiltonian of the LMG model by Eq. (D5) is shown in Fig. 6(a). The saddle point is at $\left(q^{*}, p^{*}\right)=(\pi, 0)$. We consider an initial state at $|Q=\pi, P=0\rangle$. We let it evolve according to the second quantized Hamiltonian and the mean-field Hamiltonian, respectively. To compare them, we compute the expectation values,

$$
\langle\hat{A}(t)\rangle=\left\langle Q=\pi, \quad P=0\left|e^{i \hat{H} t} \hat{A} e^{-i \hat{H} t}\right| Q=\pi, \quad P=0\right\rangle,
$$

where $\hat{A}$ is either $\hat{Q}$ or $\hat{P}$. We finally compute the difference,

$$
\Delta(t)=\sqrt{[q(t)-\langle\hat{Q}(t)\rangle]^{2}+[p(t)-\langle\hat{P}(t)\rangle]^{2}}
$$

where $[q(t), p(t)]$ is the classical trajectory beginning with $\left[q(0)=q^{*}-2 \pi / L, \quad p(0)=p^{*}\right]$. This little shift is necessary because the saddle point is a fixed point for the classical dynamics. The time evolution of $\Delta(t)$ is shown in Fig. 6(b) where we find that before the finite time $t_{E} \approx 1.5$ it is close to zero and it grows rapidly after that. In this sense, $t_{E}$ is the Ehrenfest time for this saddle point. In the main text, our discussion of the exponential growth of the OTOC is before this characteristic time.

\section{APPENDIX E: INVERTED HARMONIC OSCILLATOR}

The Hamiltonian of an inverted harmonic oscillator reads

$$
H(q, p)=\frac{1}{2}\left(p^{2}-q^{2}\right)
$$

By the same analysis above, it has a saddle point at $\left(q^{*}, p^{*}\right)=$ $(0,0)$ with energy 0 , whose Lyapunov exponent is $\lambda_{C}=1$. In the quantum regime, the dynamics of the wave function obeys the Schrödinger equation,

$$
i \hbar \frac{\partial \psi}{\partial t}=-\frac{\hbar^{2}}{2} \frac{\partial^{2} \psi}{\partial q^{2}}+V(q) \psi(q) .
$$

(a)

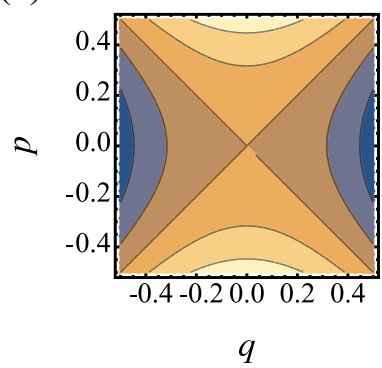

(b)

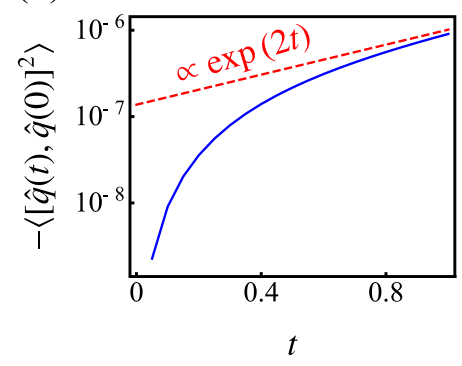

(c)

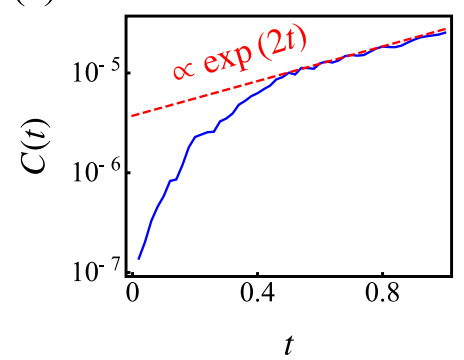

FIG. 7. Inverted harmonic oscillator with $\hbar=0.0005$. (a) The classical energy contours in phase space. A saddle point is at $\left(q^{*}, p^{*}\right)=$ $(0,0)$ with the classical Lyapunov exponent $\lambda_{C}=1$. (b) The growth of the OTOC of $-\operatorname{Tr} \hat{\rho}_{T}[\hat{q}(t), \hat{q}(0)]^{2}$ with $\hat{\rho}_{T}=e^{-\hat{H} / T} / \operatorname{Tr} e^{-\hat{H} / T}$ and $T=0.1$. (c) The growth of the OTOC of $C(t)=-\langle Q=0, P=0|[\hat{Q}(t), \hat{Q}(0)]| Q=0, P=0\rangle$. The exponential growth of these two OTOCs are both twice the classical Lyapunov exponent. 
Here we add a hard wall to the potential so that $q$ is confined by

$$
V(q)= \begin{cases}-q^{2} / 2, & q \in[-1 / 2,1 / 2], \\ \infty, & \text { otherwise. }\end{cases}
$$

This modification will not change the dynamics near the saddle point. Different from the LMG model, inverted harmonic oscillator has an infinite-dimensional Hilbert space. Numerically, we need a cutoff for momentum, which is related to the coordinate resolution. Here, we choose $\hbar=0.0005$ with the momentum cutoff at $|p| \leqslant 1=p_{\max }$, the coordinate is discretized by interval $\delta x \approx 0.0003$. The size of Planck cells is $\Delta_{q}=\sqrt{2 \pi \hbar} \approx 0.056$. This finite shift along $q$ diverges exponentially and in order to the numerical accuracy of operator and correlation function, we can only consider the time evolution at the beginning. In Figs. 7(b) and 7(c), we illustrate the growth of the OTOC of $-\left\langle[\hat{q}(t), \hat{q}(0)]^{2}\right\rangle_{T}$ and $-\left\langle Q=0, P=0\left|[\hat{Q}(t), \hat{Q}(0)]^{2}\right| Q=0, P=0\right\rangle$. We choose $T=0.1$ so that there are $80 \%$ cumulative probability of the states of energy lower than 0.05 , i.e., most contributions are made by the region near the saddle point. It is clear that the growth of these two OTOCs are both exponential with the classical Lyapunov exponent.
[1] A. I. Larkin and Y. N. Ovchinnikov, Quasiclassical method in the theory of superconductivity, JETP 28, 1200 (1969).

[2] J. Maldacena, S. H. Shenker, and D. Stanford, A bound on chaos, J. High Energy Phys. 08 (2016) 106.

[3] K. Hashimoto, K. Murata, and R. Yoshii, Out-of-time-order correlators in quantum mechanics, J. High Energy Phys. 10 (2017) 138.

[4] X. Chen and T. Zhou, Operator scrambling and quantum chaos (2018), arXiv:1804.08655.

[5] D. Bagrets, A. Altland, and A. Kamenev, Power-law out of time order correlation functions in the syk model, Nucl. Phys. B 921, 727 (2017).

[6] A. Bohrdt, C. B. Mendl, M. Endres, and M. Knap, Scrambling and thermalization in a diffusive quantum many-body system, New J. Phys. 19, 063001 (2017).

[7] J. Maldacena and D. Stanford, Remarks on the SachdevYe-kitaev model, Phys. Rev. D 94, 106002 (2016).

[8] I. García-Mata, M. Saraceno, R. A. Jalabert, A. J. Roncaglia, and D. A. Wisniacki, Chaos Signatures in the Short and Long Time behavior of the Out-of-Time Ordered Correlator, Phys. Rev. Lett. 121, 210601 (2018).

[9] E. B. Rozenbaum, S. Ganeshan, and V. Galitski, Lyapunov Exponent and Out-of-Time-Ordered Correlator's Growth Rate in a Chaotic System, Phys. Rev. Lett. 118, 086801 (2017).

[10] J. Chávez-Carlos, B. López-del Carpio, M. A. BastarracheaMagnani, P. Stránský, S. Lerma-Hernández, L. F. Santos, and J. G. Hirsch, Quantum and Classical Lyapunov Exponents in Atom-Field Interaction Systems, Phys. Rev. Lett. 122, 024101 (2019).

[11] R. A. Jalabert, I. García-Mata, and D. A. Wisniacki, Semiclassical theory of out-of-time-order correlators for low-dimensional classically chaotic systems, Phys. Rev. E 98, 062218 (2018).

[12] J. S. Cotler, D. Ding, and G. R. Penington, Out-of-time-order operators and the butterfly effect, Ann. Phys. (NY) 396, 318 (2018).

[13] T. Xu, T. Scaffidi, and X. Cao, Does Scrambling Equal Chaos? Phys. Rev. Lett. 124, 140602 (2020).

[14] K. Hashimoto, K.-B. Huh, K.-Y. Kim, and R. Watanabe, Exponential growth of out-of-time-order correlator without chaos: inverted harmonic oscillator, J. High Energy Phys. 11 (2020) 68.

[15] V. Arnold, K. Vogtmann, and A. Weinstein, Mathematical Methods of Classical Mechanics, Graduate Texts in Mathematics (Springer, New York, 2013).
[16] A. Lichtenberg and M. Lieberman, Regular and Chaotic Dynamics, Applied Mathematical Sciences (Springer, New York, 2013).

[17] L. Reichl, The Transition to Chaos: Conservative Classical Systems and Quantum Manifestations, Institute for Nonlinear Science (Springer, New York, 2013).

[18] O. Bohigas, M. J. Giannoni, and C. Schmit, Characterization of Chaotic Quantum Spectra and Universality of Level Fluctuation Laws, Phys. Rev. Lett. 52, 1 (1984).

[19] K. Takahashi and N. Saitô, Chaos and Husimi Distribution Function in Quantum Mechanics, Phys. Rev. Lett. 55, 645 (1985).

[20] H. Korsch and M. Berry, Evolution of wigner's phase-space density under a nonintegrable quantum map, Physica D 3, 627 (1981).

[21] X. Han and B. Wu, Entropy for quantum pure states and quantum $h$ theorem, Phys. Rev. E 91, 062106 (2015).

[22] D. Zhang, H. T. Quan, and B. Wu, Ergodicity and mixing in quantum dynamics, Phys. Rev. E 94, 022150 (2016).

[23] S. Moudgalya, T. Devakul, C. W. von Keyserlingk, and S. L. Sondhi, Operator spreading in quantum maps, Phys. Rev. B 99, 094312 (2019).

[24] Z. Wang, Y. Wang, and B. Wu, Quantum chaos and physical distance between quantum states, Phys. Rev. E 103, 042209 (2021).

[25] A. Nahum, S. Vijay, and J. Haah, Operator Spreading in Random Unitary Circuits, Phys. Rev. X 8, 021014 (2018).

[26] J. Jiang, Y. Chen, and B. Wu, Quantum ergodicity and mixing and their classical limits with quantum kicked rotor, arXiv: 1712.04533 .

[27] C. Yin, Y. Chen, and B. Wu, Wannier basis method for the kolmogorov-arnold-moser effect in quantum mechanics, Phys. Rev. E 100, 052206 (2019).

[28] Y. Fang, F. Wu, and B. Wu, Quantum phase space with a basis of wannier functions, J. Stat. Mech.: Theory Exp. (2018) 023113.

[29] J. von Neumann, Beweis des ergodensatzes und desh-theorems in der neuen mechanik, Z. Phys. 57, 30 (1929).

[30] J. von Neumann, Proof of the ergodic theorem and the htheorem in quantum mechanics, Eur. Phys. J. H 35, 201 (2010).

[31] R. J. Lewis-Swan, A. Safavi-Naini, J. J. Bollinger, and A. M. Rey, Unifying scrambling, thermalization and entanglement through measurement of fidelity out-of-time-order correlators in the dicke model, Nat. Commun. 10, 1581 (2019). 
[32] E. B. Rozenbaum, L. A. Bunimovich, and V. Galitski, Early-Time Exponential Instabilities in Nonchaotic Quantum Systems, Phys. Rev. Lett. 125, 014101 (2020).

[33] J. J. Sakurai, Modern Quantum Mechanics, edited by S. F. Tuan (Addison-Wesley, Boston, MA, 1985).

[34] N. Bleistein and R. Handelsman, Asymptotic Expansions of Integrals, Dover Books on Mathematics Series (Courier Corporation, North Chelmsford, MA, 1986).

[35] D. L. Shepelyanskii, Dynamical stochasticity in nonlinear quantum systems, Theor. Math. Phys. 49, 925 (1981).

[36] Z. P. Karkuszewski, J. Zakrzewski, and W. H. Zurek, Breakdown of correspondence in chaotic systems: Ehrenfest versus localization times, Phys. Rev. A 65, 042113 (2002).

[37] Y. Zhao and B. Wu, Quantum-classical correspondence in integrable systems, Sci. China Phys. Mech. Astron. 62, 997011 (2019).

[38] Z. Hu, Z. Wang, and B. Wu, Generalized wigner-von neumann entropy and its typicality, Phys. Rev. E 99, 052117 (2019).

[39] B. Chirikov and D. Shepelyansky, Chirikov standard map, Scholarpedia 3, 3550 (2008), revision No. 194619.
[40] E. J. Heller, Quantum localization and the rate of exploration of phase space, Phys. Rev. A 35, 1360 (1987).

[41] L. Fonda, G. C. Ghirardi, and A. Rimini, Decay theory of unstable quantum systems, Rep. Prog. Phys. 41, 587 (1978).

[42] X. Han and B. Wu, Ehrenfest breakdown of the mean-field dynamics of bose gases, Phys. Rev. A 93, 023621 (2016).

[43] S. Pilatowsky-Cameo, J. Chávez-Carlos, M. A. BastarracheaMagnani, P. Stránský, S. Lerma-Hernández, L. F. Santos, and J. G. Hirsch, Positive quantum Lyapunov exponents in experimental systems with a regular classical limit, Phys. Rev. E 101, 010202(R) (2020).

[44] B. Wu and J. Liu, Commutability between the Semiclassical and Adiabatic Limits, Phys. Rev. Lett. 96, 020405 (2006).

[45] L. G. Yaffe, Large $n$ limits as classical mechanics, Rev. Mod. Phys. 54, 407 (1982).

[46] J. Fröhlich, S. Graffi, and S. Schwarz, Mean-field- and classical limit of many-body schrödinger dynamics for bosons, Commun. Math. Phys. 271, 681 (2007).

[47] S. Pappalardi, A. Russomanno, B. Žunkovič, F. Iemini, A. Silva, and R. Fazio, Scrambling and entanglement spreading in longrange spin chains, Phys. Rev. B 98, 134303 (2018). 